\title{
Diagnosis and Treatment of Low Intracranial Pressure Hydrocephalus
}

\author{
Xuejian Wang*, Zhifeng Wang, Yang Chen \\ Department of Neurosurgery, The Second Hospital Affiliated to Nantong University, Nantong University, Nantong, PR China \\ Email address: \\ 6841441@163.com (Xuejian Wang) \\ ${ }^{*}$ Corresponding author \\ To cite this article: \\ Xuejian Wang, Zhifeng Wang, Yang Chen. Diagnosis and Treatment of Low Intracranial Pressure Hydrocephalus. International Journal of \\ Neurologic Physical Therapy. Vol. 3, No. 4, 2017, pp. 35-37. doi: 10.11648/j.ijnpt.20170304.13
}

Received: March 20, 2017; Accepted: April 10, 2017; Published: October 23, 2017

\begin{abstract}
Objective: To explore the clinical symptoms, diagnosis and treatment of low intracranial pressure hydrocephalus (LPH). Methods: The ventriculo-peritoneal shunt (VPS) with pressure-adjustable valves was performed in 5 patients with LPH. The diagnosis of LPH in 2 patient was definitely made by lumbar puncture drainage before VPS. LPH developed in other 3 patients with normal pressure hydrocephalus during the following-up which the patients' symptoms were not improved. Results: The following-up from 3 to 31 months after VPS showed that the clinical symptoms were improved and the enlarged ventricles significantly retracted in all patients. Conclusions: LPH is uncommon and occultly comes on. LPH is similar to normal pressure hydrocephalus in the clinical manifestation. CT and MRI show that there is ventriculomegaly in all the patients with LPH and periventricular edema in some patients with LPH. VPS with pressure-adjustable valve is an effective method to treat LPH. The perfect threshold value of pressure adjustable valves, which is suitable for the individual, may be got by more than once adjustment and long-term observation of the curative effect.
\end{abstract}

Keywords: Low Pressure Hydrocephalus(LPH), Ventriculo-Peritoneal Shunt, Pressure-Adjustable Valves

\section{Introduction}

Low intracranial pressure hydrocephalus (LPH) is uncommon and occultly comes on. LPH is similar to normal pressure hydrocephalus in the clinical manifestation. Patients share signs and symptoms such as low level of consciousness, headaches, vomiting, and cranial neuropathies [1, 2, 3], accompanying with ventriculomegaly and periventricular edema in iconography. In present, it was reported in sporadic case.

\section{Method}

Ethics statement. All study was conducted according to the principles expressed in the Declaration of Helsinki. The Ethical Committee of the Second Hospital affiliated to Nantong University has specifically approved this study. (2016002)

\subsection{Patients and Variables}

A retrospective cohort study was performed using the intern electronic health record from our hospital. From October 2010 to January 2013, a total of 5 consecutive patients were treated for LPH. Three are man, two women; The mean age was 61 years old (min, 44; max, 73). Course of disease was in five months to four years. All five patients developed ventriculomegaly: two following subarachnoid hemorrhage; one after intraventricular hemorrhage; two after cerebral trauma. The diagnosis of LPH in 2 patient was definitely made by lumbar puncture drainage before VPS. LPH developed in other 3 patients with normal pressure hydrocephalus during the following-up which the patients' symptoms were not improved.

\subsection{Clinical Manifestation}

All cases show headache, a declining level of consciousness and function, with decreases in areas such as appetite, energy, speech, motor function, and balance. The clinical picture in CT and MRI is ventriculomegaly and periventricular edema which similar to high Intracerebral pressure (ICP).

\subsection{Surgical Methods}

A variable-pressure ventriculiperitoneal shunt were used in 
all five cases (Medos-Codman, Johnson \& Johnson). Two patients were definitely diagnosed as low intracranial pressure hydrocephalus (LPH) by lumbar puncture drainage before VPS, and set pressure-adjustable valve according measurement before VPS. The other three patients were diagnosed as normal intracranial pressure hydrocephalus before VPS, but their pressure was in the lower limit of normal (100mmH2O, more or less). They are all set pressure-adjustable valve according measurement before VPS. The shunt was revised repeatedly according the patient's symptoms and the degree of ventriculomegaly in CT and MRI.

\section{Result}

Five LPH patients were eligible for analysis. Median time of follow-up was 11 months (interquartile range 3.0-31.0). Two cases LPH diagnosed before shunt were adjusted $(60 \mathrm{mmH} 2 \mathrm{O}$ to $30 \mathrm{mmH} 2 \mathrm{O} ; 50 \mathrm{mmH} 2 \mathrm{O}$ to $40 \mathrm{mmH} 2 \mathrm{O})$. The other 3 patients with normal pressure hydrocephalus were developed LPH until the patients' symptoms were improved during the following-up. one patient was prompted negative intracranial pressure hydrocephalus in the postoperative lumbar puncture; another case of LPH diagnosed after VPS (regulator for recurrence of symptoms until down to 30 $\mathrm{mmH} 2 \mathrm{O}$, and thus confirmed) met orthostatic dizziness and fatigue after leaving hospital one month later; it's brain CT scan showed narrow intraventricular, and symptoms improved after pressure valve was adjusted to $50 \mathrm{mmH} 2 \mathrm{O}$. CT and MRI in all five cases show that ventriculomegaly becomes to normal more or less and periventricular edema is alleviated.

\section{Discussion}

In 1965, Adams et al [4] first reported the phenomenon of normal intracranial pressure hydrocephalus, and named it. It was subsequently reported $[2,5]$ the phenomenon of lower intracranial pressure hydrocephalus. Some authors often confused the two as a concept. Until 1994, Pang et al [1] first proposed LPH; then more recently by Owler [6]. It was a rare clinical entity, and has been reported in sporadic cases.

At present, the mechanism of low pressure hydrocephalus remains unclear. Pang et al [1] proposed that in a small part of patients with hydrocephalus, neurologic function relates directly to ventricular size and not to intraventricular pressures. They also raised a new concept, which they think that LPH is related from its change of viscoelastic properties of brain and it was more susceptible populations for LPH in those innate low flexibility of the brain tissue than normal. Filippidis et al [7] proposed that in some cases the establishment of a transmantle pressure due to a CSF leak and a block in the communication between the ventricles and the subarachnoid space could initiate the development of the clinical entity. Changes in brain turgor follow and feed the phenomenon of LPH. In addition, due to the long-term hydrocephalus, nature of cerebrospinal fluid changed with the chronic development of hyperplasia in periventricular glial cell, may potentially change flexibility of brain which can cause LPH.
Currently, low intracranial pressure hydrocephalus are classified in the normal intracranial pressure hydrocephalus. According to clinical, radiologic, and treatment of the low-pressure hydrocephalus, we believe that it is wrong to confuse it, which needs attention. Symptoms of high intracranial pressure and ventriculomegaly were present, although the measured pressures were low, or even negative in a few case. Diagnosis of LPH is always accompanied with the process of the treatment from beginning to end, and requires to observe symptoms in a long-term with imaging studies repeatedly to adjust the height of ventricular drainage until finding a valve pressure in which point hydrocephalus symptoms could improve and no ventricle morphology repeated, and whose pressure values below the normal range of intracranial pressure, in order to make a diagnosis.

After reviewing diagnosis and treatment of this 5 cases LPH, and studying the past literature, we believe that the following points need to be considered for the diagnosis of low intracranial pressure hydrocephalus: a, intracranial pressure was in range of low or normal or normal-low; b, CT and MRI imaging show performance of hydrocephalus, such as: enlarged ventricles, periventricular edema and so on; c, how to change in clinical and image after adjustment the height of ventricular drainage device; $\mathrm{d}$, clinical and radiographic findings were improved after negative ventricular suction drainage, but the symptoms was recurrence after drainage pressure rose; e, No symptoms of overdrainage were recorded in these patients; f, neurological function was delayed decline after symptoms of VPS was relief in short-term, exclude excessive drainage from imaging findings and brain atrophy in the elderly, and repeated observations of the regulator until the threshold of pressure valve is set lower than normal intracranial pressure in same age.

Pang et al [1] suggest that negative ventricular suction drainage was used in cases which were diagnosed of low pressure hydrocephalus to promote ventricle morphology lessen, symptoms improved, but this increase the chances of infection, is unknown whether the patient can be eventually extubated or tolerated after extubated the original shunt minimum pressure setting established before. According to our experience, we need take pressure adjustable valves treatments as far as possible for the preoperative normal-low pressure hydrocephalus, which can repeatedly was adjusted but no invasion in vitro according patient's condition and get treatment opportunities when met a LPH. The initial pressure setting should be based on the measured pressure, set in a relatively high threshold, and then be gradually reduced to the desired pressure every week or so in $10-20 \mathrm{mmH} 2 \mathrm{O}$ [8]. The threshold should be moderate between intracranial pressure and the valve, too small $(0-10 \mathrm{mmH} 2 \mathrm{O})$ to shunt insufficient, too large $(>50 \mathrm{mmH} 2 \mathrm{O}$ ) lead to shunt excessive risk. Clarke MJ et al[9] proposed that after the negative ventricular pressure drainage or VPS pressure setting postoperative, long-term outcome is poor in LPH patients whose ventricle cannot fastly retracted and rapid developed. The pressure value setting depends on ventricle size and symptoms, compare with brain CT or MRI and combine of the patient's 
symptoms each time, excluding the blockage of drainage tube, brain atrophy and other organic disease or physiological and psychological factors, and highly suspect symptoms of hydrocephalus before considering the pressure value is set too high. [10, 11, 12] 5 patients were treated in our department and effect in following up for 3 to 31 months.

In this group, one normal intracranial pressure hydrocephalus case preoperative, 70 -year-old male, decline in the ability of life after head trauma, disturbance of consciousness, decreased self-care ability. It was prompted ventricular dilatation, periventricular edema in CT and MRI. Cerebrospinal fluid protein and cell count are normal routine lumbar puncture preoperative, and cerebrospinal fluid pressure is $70 \mathrm{mmHg}$. Threshold of pressure valve was set to $60 \mathrm{mmH} 2 \mathrm{O}$. Patients consciousness gradually recovered postoperative, and can eat without vomiting. It showed ventricle tends to shrink in Review CT imaging. Without repeated symptoms again, he was cured and left hospital. Ventricle morphology is gradually retracted from CT scan in one week and one month later after VPS. The patient go see doctor for an intermittent abdominal discomfort one month later. Intracranial infection was excluded in routine lumbar puncture, but negative pressure was met in lumbar puncture. Cerebrospinal Fluid (CSF) examination results suggest that a normal CSF protein. Upon the symptomatic treatment, his abdominal pain was got better. We consider it is a case of negative pressure hydrocephalus, and continue to follow-up now. The remaining two cases which are normal pressure hydrocephalus preoperative manifested as headache, cognitive decline, abnormal emotional reactions, lethargy and other symptoms. MRI examination showed enlarged ventricles. All two cases got VPS treatment with pressure-adjustable valves, and both syndromes have been alleviated and leave hospital. One of them (confirmed as a $\mathrm{LPH}$ after pressure value was set in $30 \mathrm{mmH} 2 \mathrm{O}$ and syndromes was recovered) met orthostatic dizziness, fatigue, narrow ventricles in CT scan and was raised the threshold to $50 \mathrm{mmH} 2 \mathrm{O}$, then symptoms improved and discharged in good condition of living in the follow-up.

\section{Conclusion}

Currently, the literature is less reported about the etiology and mechanisms of LPH. In clinical, hydrocephalus may manifest with difficult-to-treat low-pressure hydrocephalus which of similar symptoms caused by increased ICP. It also needs for further clinical studies.

\section{Abbreviation List}

Low intracranial pressure hydrocephalus (LPH)

Ventriculo-peritoneal shunt (VPS)

Computed Tomography CT)

Computed Tomography (MRI)

Intracerebral pressure (ICP).

\section{Cerebrospinal Fluid (CSF)}

\section{Acknowledgements}

This research supported by: (1) Young Medical Project of Nantong City Health Bureau (WQ2014016); (2) Traditional Chinese medicine science and technology project in Jiangsu province (No. YB2015113); (3) the science and technology program of Nantong city (no.: MS12015016).

\section{References}

[1] Pang D, Altschuler E. Low-pressure hydrocephalic state and viscoelastic alterations in the brain. Neurosurgery.1994 Oct; 35(4):643-55; discussion 655-6.

[2] Singounas EG, Krasanakis C, Karvounis PC. Observations on the pathogenesis of low pressure hydrocephalus. Analysis of 25 cases. Neurochirurgia (Stuttg).1976 Jan; 19(1):22-5.

[3] Vassilyadi M, Farmer JP, Montes JL. Negative-pressure hydrocephalus. J Neurosurg.1995 Sep; 83(3):486-90.

[4] Adams RD, Fisher CM, Hakim S, Ojemann RG, Sweet WH. Symptomatic occult hydrocephlus with "normal" cerebrospinal-fluid pressure: a treatable syndrome [J]. N Engl J Med. 1965 Jul 15; 273:117-26.

[5] Ingram TT. 'Low-pressure hydrocephalus'. Dev Med Child Neurol.1971 Oct; 13(5):676.

[6] Owler BK, Jacobson EE, Johnston IH. Low pressure hydrocephalus: issues of diagnosis and treatment in five cases. Br J Neurosurg.2001 Aug;15(4):353-9.

[7] Filippidis AS, Kalani MY, Nakaji P, Rekate HL. Negative-pressure and low-pressure hydrocephalus: the role of cerebrospinal fluid leaks resulting from surgical approaches to the cranial base. J Neurosurg.2011 Nov; 115(5):1031-7. doi: 10.3171/2011.6. JNS101504. Epub 2011 Jul 29.

[8] Zemack G, Romner B. Adjustable valves in normal-pressure hydrocephalus: a retrospective study of 218 patients. Neurosurgery.2002 Dec; 51(6):1392-400; discussion 1400-2.

[9] Michelle J, Clarke, Cormac O, et al. Very low pressure hydrocephalus: report of two cases [J]. J Neurosurg, 2006, 105(3): 475-478.

[10] Sato H, Koizumi T, Sato D, Endo S, Kato S. Unilateral Posterior Reversible Encephalopathy Syndrome after Ventriculo-Peritoneal Shunt for Normal Pressure Hydrocephalus Following Subarachnoid Hemorrhage: A Case Report. No Shinkei Geka. 2016 Jun; 44(6):507-15.

[11] Takahashi Y, Kawanami T, Nagasawa H, Iseki C, Hanyu H, Kato T. Familial normal pressure hydrocephalus (NPH) with an autosomal-dominant inheritance: a novel subgroup of NPH. J Neurol Sci. 2011 Sep 15; 308(1-2):149-51.

[12] Cheng Z, Wang W, Han Y, Liu J, Wang Z, Zhou Y, Li F, Huang Y. Low pressure hydrocephalus: clinical manifestations, radiological characteristics, and treatment. Br J Neurosurg. 2017 Mar 6:1-5. 